\title{
The Steel Equivalency Workbook: An X-Ray Transmission Calculator
}

Zachary J. Thompson

Asa G. Monson

Robert D. Seifert

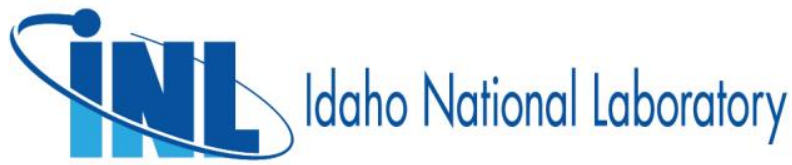




\section{DISCLAIMER}

This information was prepared as an account of work sponsored by an agency of the U.S. Government. Neither the U.S. Government nor any agency thereof, nor any of their employees, makes any warranty, expressed or implied, or assumes any legal liability or responsibility for the accuracy, completeness, or usefulness, of any information, apparatus, product, or process disclosed, or represents that its use would not infringe privately owned rights. References herein to any specific commercial product, process, or service by trade name, trade mark, manufacturer, or otherwise, does not necessarily constitute or imply its endorsement, recommendation, or favoring by the U.S. Government or any agency thereof. The views and opinions of authors expressed herein do not necessarily state or reflect those of the U.S. Government or any agency thereof. 
INL/EXT-21-64582

Revision 000

\title{
The Steel Equivalency Workbook: An X-Ray Transmission Calculator
}

\author{
Zachary J. Thompson \\ Asa G. Monson \\ Robert D. Seifert
}

September 2021

\begin{abstract}
Idaho National Laboratory
Idaho Falls, Idaho 83415
\end{abstract}

http://www.inl.gov

Prepared for the Project Manager

Recovered Chemical Materiel Directorate, United States Army

Under DOE Idaho Operations Office

Contract DE-AC07-05ID14517 
Page intentionally left blank 


\section{SUMMARY}

The Steel Equivalency Spreadsheet was created as a robust, user-friendly method for:

1. Determining if it is possible to image an object.

2. Reducing time needed when selecting equipment and preparing for field imaging activities.

3. Reducing the amount of equipment taken for field imaging activities.

4. Determining a starting point for exposure settings prior to imaging an object.

5. Reducing the amount of dose deposited to complete an imaging operation (in the spirit of As Low As Reasonably Achievable, ALARA).

The spreadsheet was developed using various physical models to account for different phenomena. Future implementations aim to expand beyond the Digital Radiography and Computed Tomography Single Munition Scanner (DRCT SMS) in standard configuration to include high energy XRGs (Betatrons) and sub-MeV pulsed XRGs (XRS4). Alternative computational methods that compensate for incoherent scattering when calculating relative transmission are also being pursued. Ultimately the spreadsheet exceeded the developmental goal of having less than $10 \%$ average error when comparing calculations to real-world images. 
Page intentionally left blank 


\section{ACKNOWLEDGEMENTS}

A special thanks for their guidance, support, and suggestions goes out to (in alphabetical order by last name):

Stacey Barker, INL

Russell (Rusty) Fendick, US Army, CMA, RCMD (retired)

Gerald (Jerry) Godwin, US Army, 20th CBRNE Command, CARA

Charles Maddox, US Army, 20th CBRNE Command, CARA

James Milloway, INL

Bruce Peter, US Army, 20th CBRNE Command, CARA

Yancey Rhoden, US Army, 20th CBRNE Command, CARA

Tim Roney, INL (Retired)

Michael Rowan, US Army, 20th CBRNE Command, CARA

Mike Smith, ISU, IAC 
Page intentionally left blank 


\section{CONTENTS}

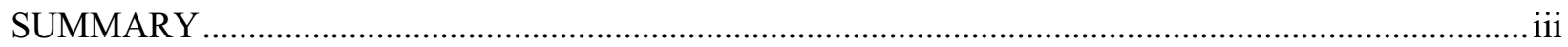

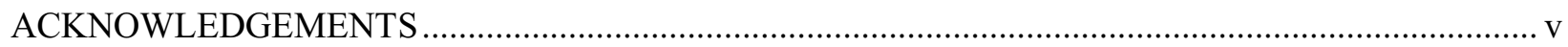

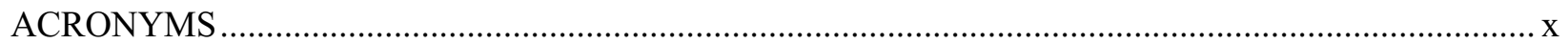

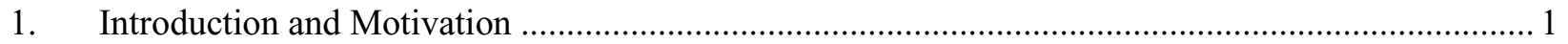

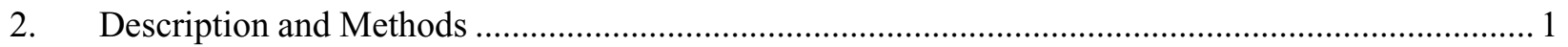

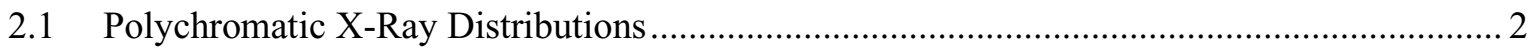

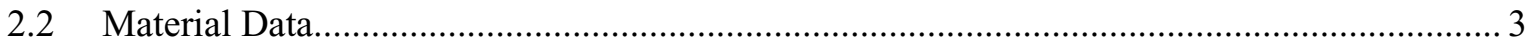

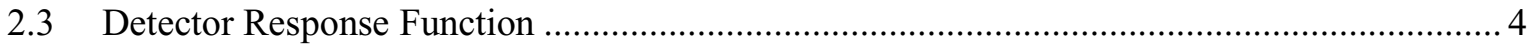

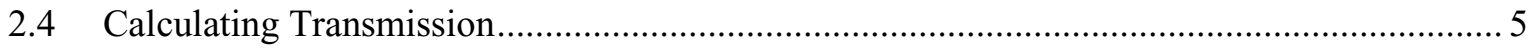

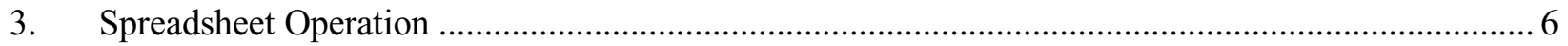

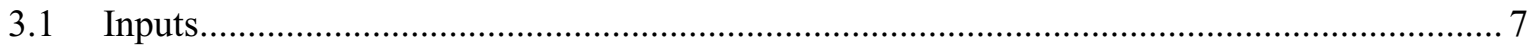

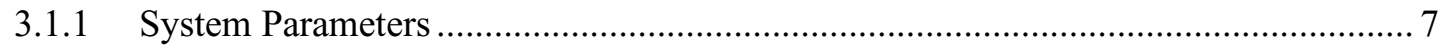

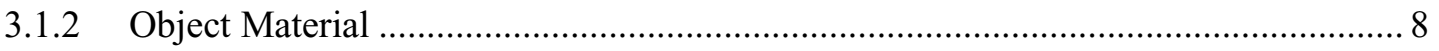

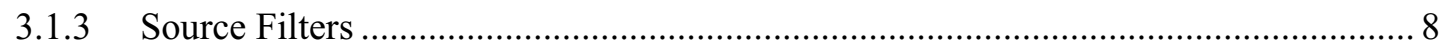

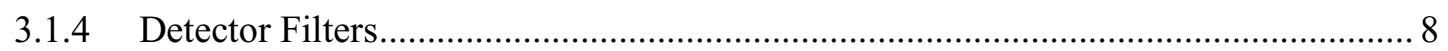

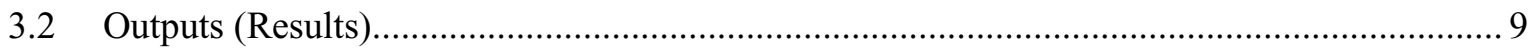

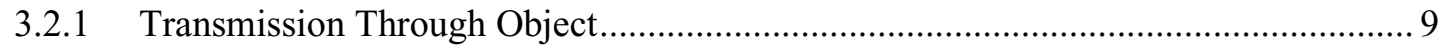

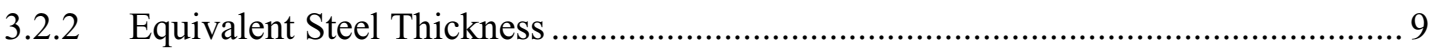

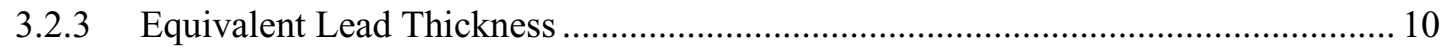

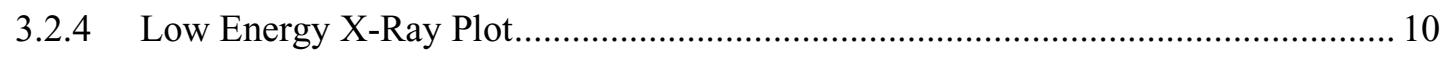

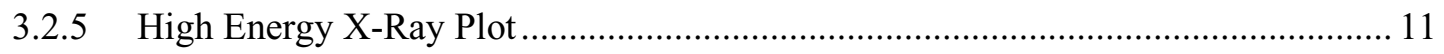

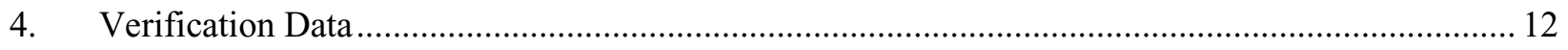

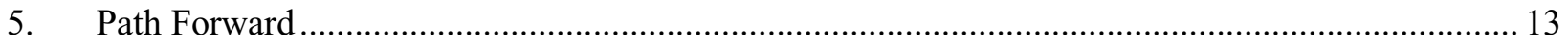

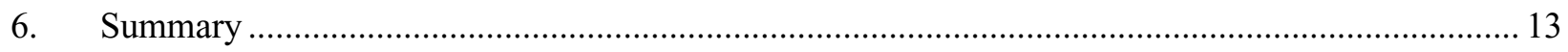

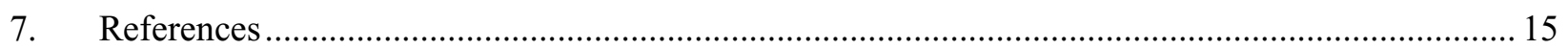

FIGURES

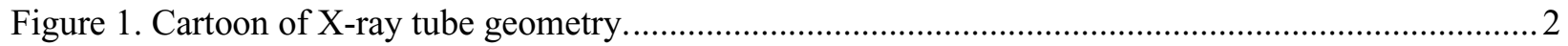

Figure 2. Simulated X-ray energy spectra at different voltage biases. ................................................ 3

Figure 3. Example mass attenuation as a function of photon energy for tungsten (W). Note that minor artifacts are present near $10 \mathrm{keV}$ due to the PCHIP spline. ............................................ 4

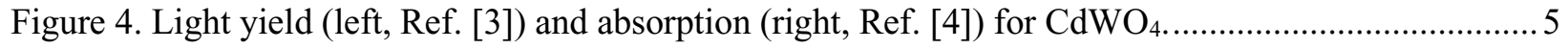

Figure 5. Front page of the Steel Equivalency Calculator. ...................................................................... 7 
Figure 6. Examples of correct system parameters (left) and incorrect (right). The maximum allowed current for the selected XRG is $3.0 \mathrm{~mA}$.

Figure 7. Example object composition. All dimensions are in inches.................................................... 8

Figure 8. Example source filter stack. All dimensions are in inches. ...................................................... 8

Figure 9. Example detector filter stack. All dimensions are in inches................................................ 9

Figure 10. Example output for the relative transmission, equivalent steel thickness, and equivalent

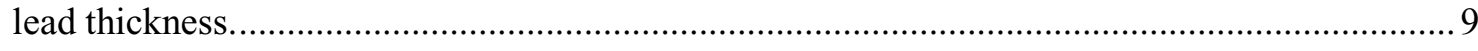

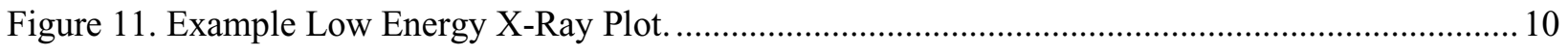

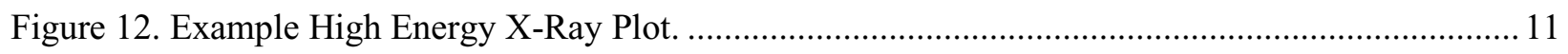

Figure 13. Cropped DR of the steel stepwedge used for verification. .................................................. 12

\section{TABLES}

Table 1. Relative transmission from a DR compared to calculations. 12 
Page intentionally left blank 


\section{ACRONYMS}

$\begin{array}{ll}\text { ALARA } & \text { As Low As Reasonably Achievable } \\ \text { CARA } & \text { CBRNE Analytical and Remediation Activity } \\ \text { CBRNE } & \text { Chemical, Biological, Radiological, Nuclear, Explosive } \\ \text { CMA } & \text { Chemical Materials Activity } \\ \text { CR } & \text { Computed Radiography } \\ \text { DDA } & \text { Direct Digital Array } \\ \text { DR } & \text { Digital Radiograph } \\ \text { DRCT } & \text { Digital Radiography and Computed Tomography } \\ \text { DRCT SMS } & \text { Digital Radiography and Computed Tomography Single Munition Scanner } \\ \text { DT } & \text { Detection Technologies } \\ \text { GUI } & \text { Graphical User Interface } \\ \text { HPC } & \text { High Performance Computing } \\ \text { IAC } & \text { Idaho Accelerator Center } \\ \text { INL } & \text { Idaho National Laboratory } \\ \text { ISU } & \text { Idaho State University } \\ \text { LDA } & \text { Linear Diode Array } \\ \text { LIXS } & \text { Large Item X-Ray Scanner } \\ \text { NIST } & \text { National Institute of Standards and Technology } \\ \text { PENELOPE } & \text { Penetration and Energy Loss of Positrons and Electrons } \\ \text { PCHIP } & \text { Piecewise Cubic Hermite Interpolating Polynomial } \\ \text { RCMD } & \text { Recovered Chemical Materiel Directorate } \\ \text { SDS } & \text { Safety Data Sheet } \\ \text { TCXT } & \text { Ton Container X-Ray Trailer } \\ \text { XML } & \text { Extensible Markup Language } \\ \text { XRG } & \text { X-Ray Generator } \\ & \end{array}$


Page intentionally left blank 


\section{The Steel Equivalency Workbook: An X-Ray Transmission Calculator}

\section{Introduction and Motivation}

Determining appropriate X-ray equipment for an imaging situation is a daunting task. Past methodologies rely on prior imaging experience for this determination. However, even skilled radiographers may have issues choosing proper equipment. A new, more robust method was required with the aim to:

1. Determine if it is possible to image an object.

2. Reduce time needed when selecting equipment and preparing for field imaging activities.

3. Reduce the amount of equipment taken for field imaging activities.

4. Determine a starting point for exposure settings prior to imaging an object.

5. Reduce the amount of dose deposited to complete an imaging operation (in the spirit of As Low As Reasonably Achievable, ALARA).

The Steel Equivalency Spreadsheet was developed to facilitate this goal. This effort focused on creating a model for the Digital Radiography and Computed Tomography Single Munition Scanner (DRCT SMS) in standard configuration, equipped with a Yxlon Smart 300 HP X-ray generator (XRG) and Detection Technologies (DT) linear diode array (LDA). After a suitable model was created, other XRGs and other detectors were added in an attempt to implement the full complement of deployable assessment equipment for the Recovered Chemical Materiel Directorate (RCMD).

The motivation for the author to create a tool for modeling X-ray transmission through materials involved the Chemical, Biological, Radiological, Nuclear, Explosive (CBRNE) Analytical and Remediation Activity (CARA) personnel developing tables of half-value layers for various materials to aid in selecting XRG and detector combinations. The measurement of half-value layers, while useful, does not fully capture the relationship between XRG output, material absorption, and measured signal. An improved method was required. A path forward was determined based on an extensive literature review and discussions with field and laboratory radiography experts.

\section{Description and Methods}

The methods for physically describing X-ray propagation and matter interaction are well understood. However, the mathematics used to describe them are complex and are recursive like all other light-matter interactions.

In order to adequately and accurately describe X-ray transmission through a defined object, the following are required:

1. Accurate, high-fidelity models of XRGs.

2. X-ray attenuation data for materials of interest need to be compiled from reliable sources into a library.

3. Accurate modeling of the X-ray detectors.

4. Proper computation using the above quantities and approximate object composition.

Through an extensive literature search, tools for modeling XRG output energy spectra were determined and response functions of detectors were compiled. The attenuation data was readily available via National Institute for Standard and Technology's (NIST's) XCOM website, Ref. [1]. Transmission through the test object was addressed with the simplest model, Beer's law. 


\subsection{Polychromatic X-Ray Distributions}

A method for modeling the energy spectrum emitted from the XRGs that are deployed with the DRCT SMS became the primary hurdle. X-rays emitted from the constant-potential sources that are used in the DRCT SMS are polychromatic, containing a continuum of X-ray energies, due to the Bremsstrahlung (braking radiation) generation mechanism. This complicates creating simple calculators because X-rays of different energies are attenuated (linearly) at different rates.

Bremsstrahlung generation of photons, not necessarily just X-rays, occurs anytime a charged particle slows down or decelerates. It is the primary means of generating X-rays in field portable applications due to its ease of generation and inherent safety; X-rays are not emitted when the system is de-energized. Xrays are produced when ejected electrons from the filament within the X-ray tube accelerate toward the anode (target), where they impact the material and slow down. A simple cartoon of this can be seen in Figure 1. While the mechanism is simple to explain, the physics and math that describes the process requires the use of numerical methods as closed form solutions are not possible using conventional techniques.

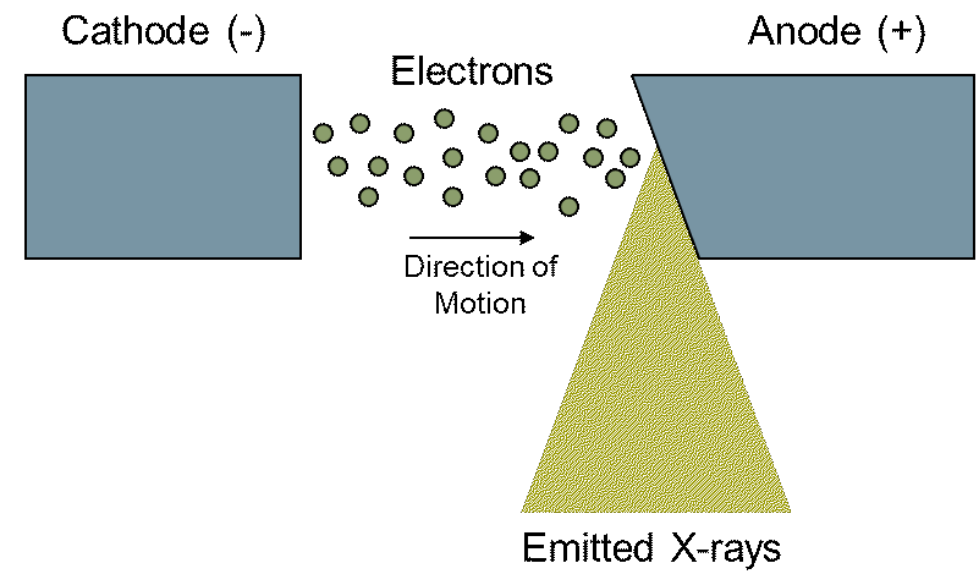

Figure 1. Cartoon of X-ray tube geometry.

The Yxlon Smart 300 HP was the focus of the modeling effort due to its use on the DRCT SMS. The approximate internal geometry of this X-ray generator is relatively simple (Figure 1) and lends itself to modeling. To first order approximation, the only parameter that drives the X-ray distribution is the voltage bias, known colloquially as $\mathrm{kV}$. In these models it was assumed that the electron beam within the XRG is monoenergetic. The conversion from voltage bias to kinetic energy of electrons within the X-ray tube is easy as clever scientists decided to define kinetic energy of electrons based on the voltage bias in which they were accelerated. To clarify, electron kinetic energy is measured in terms of the electronvolt, $\mathrm{eV}$, where $1 \mathrm{eV}$ of kinetic energy is the equivalent of one electron being accelerated in a $1 \mathrm{~V}$ potential bias. For our purposes, the voltage bias of the generator corresponds to the kinetic energy of the electrons. As an example, when a Yxlon Smart $300 \mathrm{HP}$ is set to $300 \mathrm{kV}$ it will have a stream of $300 \mathrm{keV}$ electrons impacting the target within the $\mathrm{X}$-ray tube. This was assumed because electrons have small mass, allowing them to easily reach their "terminal velocity" prior to impacting the target. The spatial distribution of the generated X-ray spectrum is not uniform. However, these variations are ignored because they slowly vary across the usable cone of radiation.

The program originally used for modeling the XRGs, PENELOPE (Penetration and ENErgy LOss of Positrons and Electrons), is a general-purpose Monte Carlo code for the simulating coupled electronphoton transport. The Python implementation of PENELOPE, pyPENELOPE, was the focus. This implementation includes its own graphical user interface (GUI). However, the GUI has input parameter limits that can be circumvented when directly editing the input extensible markup language (XML) file. 
The angle of the target and the width of the electron beam necessitated this work around. However, in practice, once the electron beam width exceeded the allowed values in the GUI, it changed little.

The modeled X-ray energy spectra as a function of energy are denoted as $\phi\left(E, E_{\max }\right)$ where $E_{\max }$ denotes the maximum energy (XRG kV setting). These spectra are normalized probability distributions, $\int_{0}^{E_{\max }} \phi\left(E, E_{\max }\right) d E=1$, that represent the probability of an electron traveling with $E_{\text {max }}$ kinetic energy creating a photon of $E$ energy through interaction with the target material. Models for voltage biases ranging from $50 \mathrm{kV}$ to $300 \mathrm{kV}$ in steps of $25 \mathrm{kV}$ were generated. These values cover the entire operational range of the Yxlon Smart 300 HP. Several of these energy spectra can be seen in Figure 2.

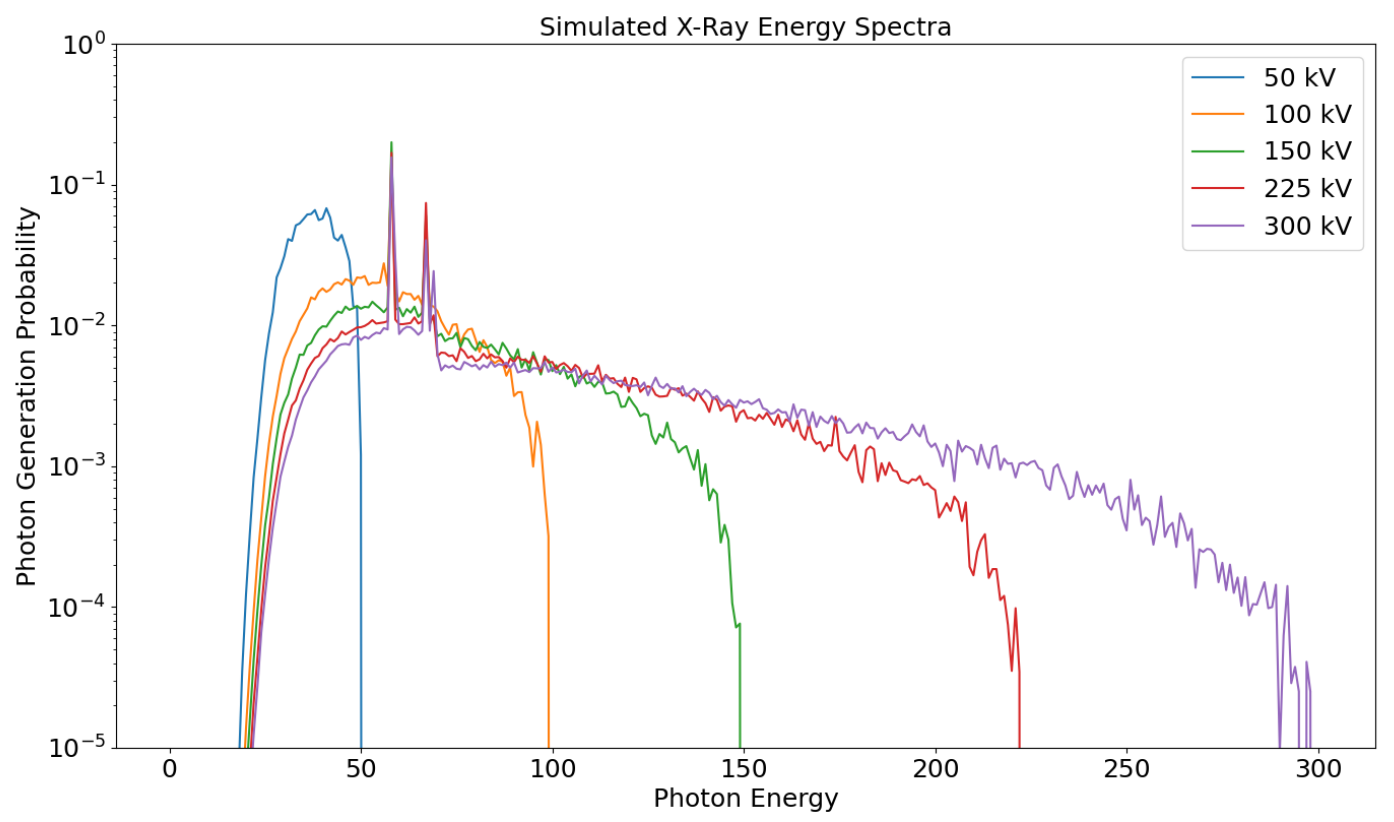

Figure 2. Simulated X-ray energy spectra at different voltage biases.

\subsection{Material Data}

The NIST XCOM database was used to compile applicable material data. This database was chosen over other NIST X-ray attenuation databases for the following reasons:

1. The modularity of acceptable inputs in terms of elements, compounds, and mixtures compared to the NIST XAAMDI database;

2. The extended energy range of the data compared to the NIST FFAST database.

Initially material data was laboriously extracted by manually entering discrete desired energy values. A python script was employed later to expedite the process. This script employs a Piecewise Cubic Hermite Interpolating Polynomial (PCHIP) spline to interpolate data between the standard output data points. A PCHIP spline was chosen because it is a shape preserving spline, ensuring that the electron shell edges $(\mathrm{K}, \mathrm{L}$, etc.) induce minimal artifacts within the data. An example of a PCHIP spline fit to the mass attenuation for tungsten is in Figure 3 (page 4).

It is important to note that the material attenuation data from NIST XCOM database is the mass attenuation (units of $\mathrm{cm}^{2} / \mathrm{g}$ ). These need to be converted to linear attenuation coefficients, which is accomplished by multiplying by the material density (units of $\mathrm{g} / \mathrm{cm}^{3}$ ). Densities for these materials were primarily harvested from the Safety Data Sheets (SDSs). 


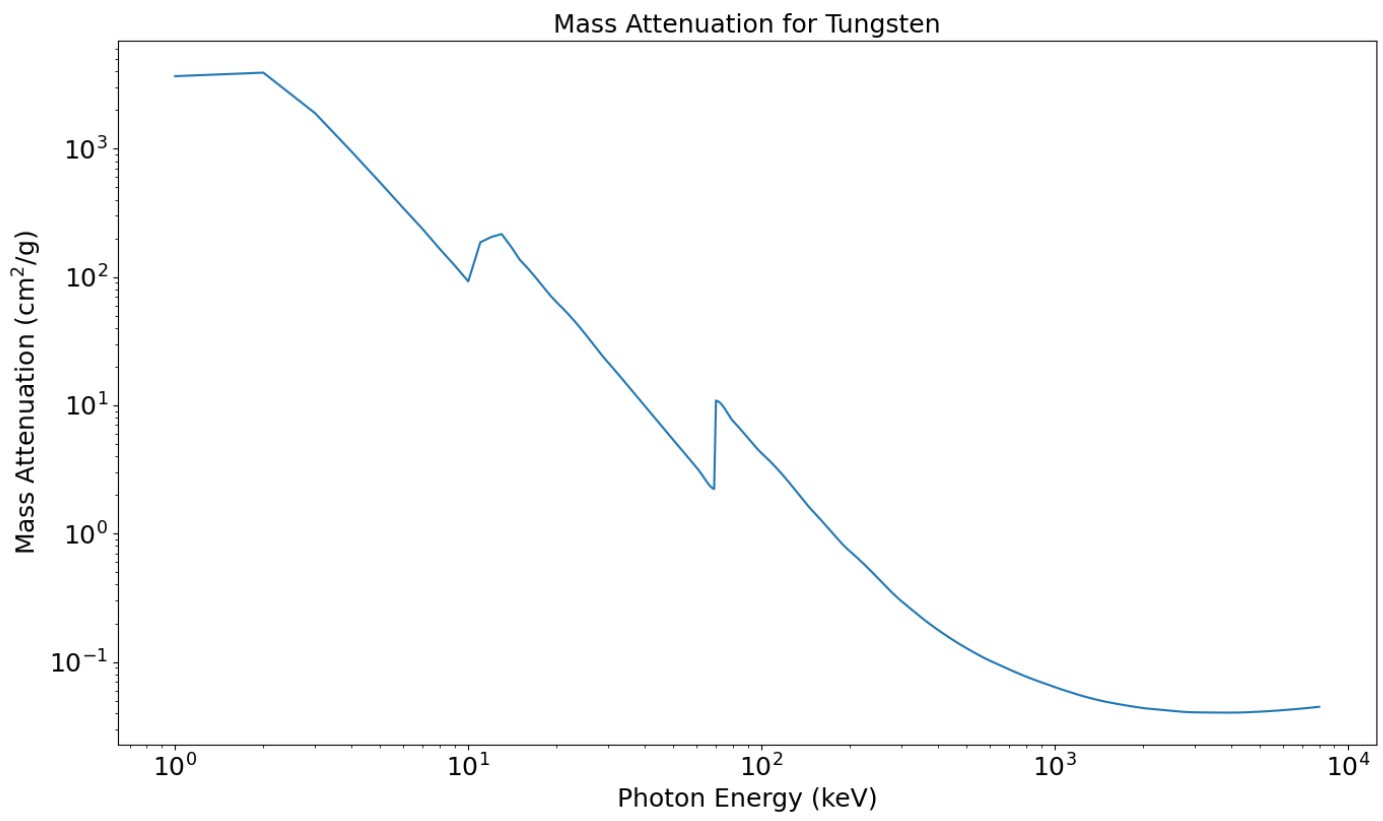

Figure 3. Example mass attenuation as a function of photon energy for tungsten (W). Note that minor artifacts are present near $10 \mathrm{keV}$ due to the PCHIP spline.

\subsection{Detector Response Function}

Modeling the detector response is a non-trivial task. Faithfully modeling the response requires creating models for each scintillation material and semiconductor photodetector combination that produces photoelectrons. These photoelectrons are then translated into a grayscale value. In order to simplify the process, it was generally assumed that the number of scintillated photons is proportional to the total signal registered on the detector.

The detector response functions were compiled through an extensive literature review. This review scoured for information based on the following assumptions:

1. The detector response, $\varepsilon(E)$, is one of two quantities:

a. $\varepsilon(E)=L(E) *\left(1-e^{-\mu(E) x}\right)$, using the absorption, $1-e^{-\mu(E) x}$, where $\mu(E)$ is the linear attenuation coefficient and $x$ is the material thickness, and the light emission response of the scintillation material $(L(E))$. The light emission response is a ratio of the number of scintillated photons per photon at a given energy divided by the number of scintillated photons per $662 \mathrm{keV}$ photon. $662 \mathrm{keV}$ is used as the standard because it is a strong gamma emission from ${ }^{137} \mathrm{Cs}$.

b. $\varepsilon(E)=G * E *\left(1-e^{-\mu(E) x}\right)$, using the absorption, $1-e^{-\mu(E) x}$, where $\mu(E)$ is the linear attenuation coefficient and $x$ is the material thickness. The detector gain $(G)$ is a fit parameter based on test images using X-ray absorptiometry (Ref. [2]).

2. All photons produced through scintillation are absorbed and contribute to the signal (pixel value). 
The detector response for the currently fielded DT iHE LDA detectors employ the former equation, where the light emission response (Figure 4, left) was taken from Ref. [3] and the absorption of the cadmium-tungstate $\left(\mathrm{CdWO}_{4}\right.$, Figure 4, right) was taken from Ref. [4]. On the other hand, the response for flat panel detectors (direct digital arrays, DDAs) require test imaging and fitting using the second method due to the lack of literature on the response of terbium doped gadolinium oxysulfide $\left(\mathrm{Tb}: \mathrm{Gd}_{2} \mathrm{O}_{2} \mathrm{~S}\right)$. This is not the only scintillation material that is employed in DDAs, however it is the one of most common.
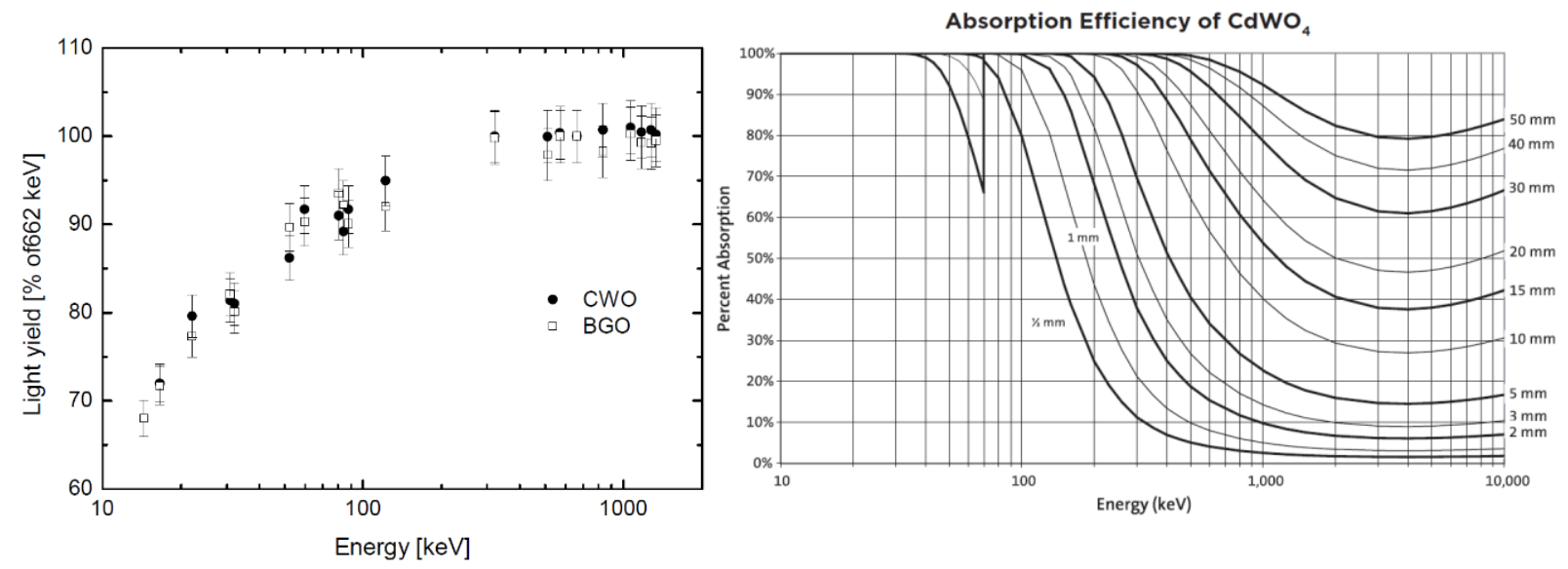

Figure 4. Light yield (left, Ref. [3]) and absorption (right, Ref. [4]) for $\mathrm{CdWO}_{4}$.

\subsection{Calculating Transmission}

In principle, a full model that incorporates all related phenomena that dissipate energy in a system is required to obtain exact results. This route is impractical due to the computational cost incurred by the required physical models for each object imaged. However, approximations and assumptions can be properly applied to simplify calculations. In this vein, relative transmission using Beer's Law was chosen to simplify computations. The general form of Beer's Law can be seen below.

$$
I=I_{0} e^{-\mu x}
$$

Eq. 1

This form of Beer's law describes the attenuation of X-rays at a given intensity, $I_{0}$, for a single material where $x$ is the material thickness and $\mu$ is the linear attenuation coefficient of that material. This formula has limited utility because it is highly unlikely to find an object of interest that is comprised of a single material. Normally these objects are complex, containing multiple materials. Luckily, this formula can be adapted to accommodate such an application.

$$
I=I_{0} e^{-\alpha}=I_{0} e^{-\sum_{i} \mu_{i} x_{i}}
$$

Eq. 2

The total attenuation at each $\alpha$, is the sum over the attenuation contributions from all the materials contained within the object. It is important to note that this will need to be applied to each energy across the entire Bremsstrahlung spectrum emitted from the XRG. More succinctly, this means the attenuated intensity at each energy must be considered.

$$
I(E)=I_{0}(E) e^{-\sum_{i} \mu_{i}(E) x_{i}}
$$

Eq. 3

Normally, the intensity is not directly measured. Instead, the integrated intensity over the Bremsstrahlung energy spectrum is measured. For a given object, the absolute measurement (pixel value, Signal $_{\text {object }}$ ) can be calculated integrating the Bremsstrahlung energy spectrum, $I(E)$, and the detector response function, $\varepsilon(E)$, over the energy range, 0 to $E_{\max }$. The detector response function $\varepsilon(E)$, discussed in Section 2.3, accounts for how efficiently the incident intensity is captured by the detector. 


$$
\text { Signal }_{\text {object }}=\int_{0}^{E_{\max }} I(E) \varepsilon(E) d E=\int_{0}^{E_{\max }} I_{0}(E) e^{-\sum_{i} \mu_{i}(E) x_{i}} \varepsilon(E) d E
$$

Absolute measurements (pixel value) are complex calculations that require modeling all parameters, including the exact geometry of an entire imaging system (x-ray generator, object, detector) and exact object composition, including voids and inhomogeneities. Pursuing a complete model was deemed an imprudent use of time and resources. Instead, the relative power transmission, $\mathrm{T}$, was chosen. Relative power transmission can be easily computed from images and doing so removes environmental factors and some system factors from the calculation. The relative power transmission takes the following form:

$$
\mathrm{T}=\frac{\text { Signal }_{\text {Object }}}{\text { Signal }_{\text {Max }}}
$$

Eq. 5

Using the definition for the Signal Object $_{\text {in }}$ Eq. 4, the relative power transmission from Eq. 5 can be re-written in the following manner:

$$
\mathrm{T}=\frac{\int_{0}^{E_{\max }} I_{0}(E) e^{-\sum_{i} \mu_{i}(E) x_{i}} \varepsilon(E) d E}{\int_{0}^{E_{\max }} I_{0}(E) \varepsilon(E) d E} \approx \frac{\int_{0}^{E_{\max }} \phi\left(E, E_{\max }\right) e^{-\sum_{i} \mu_{i}(E) x_{i}} \varepsilon(E) d E}{\int_{0}^{E_{\max }} \phi\left(E, E_{\max }\right) \varepsilon(E) d E} \quad \text { Eq. } 6
$$

Note that the denominator assumes that there is no material attenuation (i.e., an air-shot, $\mu=0$ ) for maximum signal. The progression through Eq. 6 is facilitated by assuming the intensity of the emitted Xrays from the XRG are proportional to the product of the energy spectrum $\left(\phi\left(E, E_{\max }\right)\right.$, discussed in Section 2.1) and the electrical current of the XRG during imaging. Assuming that the maximum signal and the signal from the object are collected at the same exposure settings, the contribution from the electrical current cancels.

Finally, the relative transmission can be calculated using the developed tooling. This assumes the user knows the approximate object composition so that the internal working of spreadsheet can use input values for the material thickness, $x$, and the linear attenuation coefficient of that material, $\mu$.

\section{Spreadsheet Operation}

The Steel Equivalency Calculator was designed in an Excel workbook to maximize utility. Excel provides an easily customizable platform that allows for broad distribution, does not require installation of non-standard software, and maintains small file size.

The front page of the workbook (Figure 5, page 7) is laid out for ease of use. It is intended to provide a linear process flow for inputting parameters and displaying results without increasing clutter. To this end, the front page is broken up into four quadrants. The upper left quadrant contains all system inputs and conversion calculators. The upper right quadrant displays the low energy X-ray plot. The lower left quadrant contains allowed parameters based on the XRG. The lower right quadrant contains the high energy X-ray plot. 


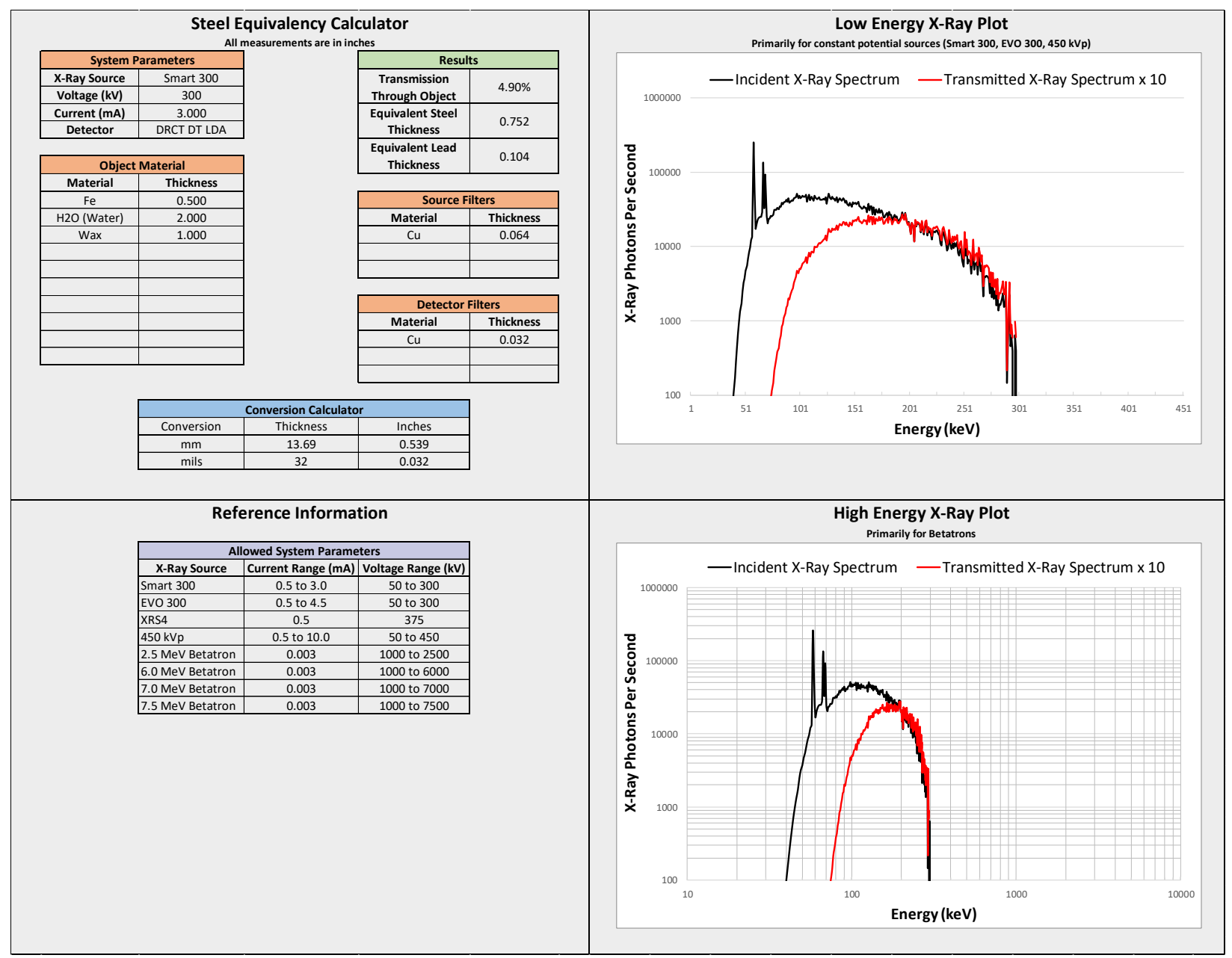

Figure 5. Front page of the Steel Equivalency Calculator.

\subsection{Inputs}

The input section of the Steel Equivalency Calculator resides in the upper left quadrant. There are specific inputs for the System Parameters, Object Material, Source Filters, and Detector Filters.

\subsubsection{System Parameters}

The system parameter window is the primary input area for the X-ray system being used. Rather than creating a drop down for each specific system, the XRG and detector are selectable so that if a nonstandard system configuration is a possibility, it can easily be employed. Allowed XRG settings are displayed in the reference information in the lower left quadrant. Failure to use these values will result in cells displaying in red. An example can be seen below in Figure 6.

\begin{tabular}{|c|c|}
\hline \multicolumn{2}{|c|}{ System Parameters } \\
\hline X-Ray Source & Smart 300 \\
\hline Voltage (kV) & 300 \\
\hline Current (mA) & 3.000 \\
\hline Detector & DRCT DT LDA \\
\hline
\end{tabular}

\begin{tabular}{|c|c|}
\hline \multicolumn{2}{|c|}{ System Parameters } \\
\hline X-Ray Source & Smart 300 \\
\hline Voltage (kV) & 300 \\
\hline Current (mA) & 4.000 \\
\hline Detector & DRCT DT LDA \\
\hline
\end{tabular}

Figure 6. Examples of correct system parameters (left) and incorrect (right). The maximum allowed current for the selected XRG is $3.0 \mathrm{~mA}$. 
The detector settings are much simpler. The user only needs to select the desired detector. This automatically populates the detector response into the appropriate sections of the calculations.

\subsubsection{Object Material}

There are ten slots for object materials (Figure 7). The approximate thickness (in inches) of the materials in an object are input into one of the ten slots. The information in this section then pulls the attenuation data for each material and calculates the transmission through the object at each energy using Beer's Law (Eq. 3).

\begin{tabular}{|c|c|}
\hline \multicolumn{2}{|c|}{ Object Material } \\
\hline Material & Thickness \\
\hline Fe & 0.500 \\
\hline H2O (Water) & 2.000 \\
\hline Wax & 1.000 \\
\hline & \\
\hline & \\
\hline & \\
\hline & \\
\hline & \\
\hline & \\
\hline & \\
\hline
\end{tabular}

Figure 7. Example object composition. All dimensions are in inches.

\subsubsection{Source Filters}

Any source filter material or series of filters needs to be populated in this section. The approximate thickness (in inches) of the filter material(s) are input into one of the three slots (Figure 8). The information in this section then pulls the attenuation information for each filter material and calculates the transmission through the filter(s) at each energy using Beer's Law (Eq. 3). The attenuation due to the filtering material will affect the X-ray energy spectra displayed in the X-Ray Plots and the "Transmission Through Object" calculation.

\begin{tabular}{|c|c|}
\hline \multicolumn{2}{|c|}{ Source Filters } \\
\hline Material & Thickness \\
\hline $\mathrm{Cu}$ & 0.064 \\
\hline & \\
\hline & \\
\hline
\end{tabular}

Figure 8. Example source filter stack. All dimensions are in inches.

\subsubsection{Detector Filters}

Any detector filter material or series of filters needs to be populated in this section. The approximate thickness (in inches) of the filter material(s) are input into one of the three slots (Figure 9, page 9). The information in this section then pulls the attenuation information for each filter material and calculates the transmission through the filter(s) at each energy using Beer's Law (Eq. 3). The "Transmission Through Object" is only location where the detector filters are used in calculations. 
Figure 9. Example detector filter stack. All dimensions are in inches.

\begin{tabular}{|c|c|}
\multicolumn{2}{|c|}{ Detector Filters } \\
\hline Material & Thickness \\
\hline $\mathrm{Cu}$ & 0.032 \\
\hline & \\
\hline & \\
\hline
\end{tabular}

\subsection{Outputs (Results)}

The output section of the Steel Equivalency Calculator resides in the upper left quadrant (Figure 5, page 7) near the input section. There are specific outputs for the Transmission Through Object, Equivalent Steel Thickness, and Equivalent Lead Thickness (see Figure 10).

\begin{tabular}{c|c|}
\multicolumn{2}{|c|}{ Results } \\
\hline $\begin{array}{c}\text { Transmission } \\
\text { Through Object }\end{array}$ & $4.90 \%$ \\
\hline $\begin{array}{c}\text { Equivalent Steel } \\
\text { Thickness }\end{array}$ & 0.752 \\
\hline $\begin{array}{c}\text { Equivalent Lead } \\
\text { Thickness }\end{array}$ & 0.104 \\
\hline
\end{tabular}

Figure 10. Example output for the relative transmission, equivalent steel thickness, and equivalent lead thickness.

\subsubsection{Transmission Through Object}

The first output is "Transmission Through Object." This is the relative transmission, expressed as a percentage, through the object. It is calculated using Eq. 6, which is commonly known as the relative integrated power transmission in optical physics research.

\subsubsection{Equivalent Steel Thickness}

The second output, "Equivalent Steel Thickness," is a comparison measurement that is a carry-over from the X-ray film and computed radiography (CR) era. The total attenuation of the object is compared to the attenuation of steel (iron). This value is calculated through the following steps:

1. Divide the total attenuation at each energy, $\alpha(E)$, by the linear attenuation coefficient for steel at that same energy, $\mu_{F e}(E)$. This yields an equivalent thickness of steel at that energy.

$$
\frac{\alpha(E)}{\mu_{F e}(E)}=x_{F e}(E)
$$

2. Take the arithmetic mean over the equivalent steel thicknesses for the applicable energy range. The applicable energy range extends from $0 \mathrm{keV}$ to $E_{\max }$. Since the data is compartmentalized into energy bins, this range runs from bin 1 to bin $n$.

$$
\frac{\sum_{1}^{n} x_{F e}(E)}{n}=\bar{x}_{F e}
$$

Eq. 8 


\subsubsection{Equivalent Lead Thickness}

The final output, "Equivalent Lead Thickness," is a comparison measurement that shows how much shielding a stack of objects constitutes. This is intended to be used for shielding calculations, this includes both engineered shielding and impromptu field applications. It is calculated in the same fashion as the equivalent "Equivalent Steel Thickness" above.

\subsubsection{Low Energy X-Ray Plot}

The Low Energy X-Ray Plot (Figure 11) is used to display the transmission through the object for the lower energy region $(0-450 \mathrm{keV})$. This region encompasses the range for systems employing constant potential XRGs such as the DRCT SMS $(0-300 \mathrm{keV})$, Large Item X-Ray System (LIXS, 0 - $300 \mathrm{keV}$ ), and Ton Container X-Ray Trailer (TCXT, $0-450 \mathrm{keV}$ ). This plot is primarily present to illustrate attenuation and beam hardening as X-rays pass through materials for the constant potential X-ray sources. The source filters are accounted for in both the incident and transmitted spectra. The detector filters are not used to calculate the spectra in this plot.

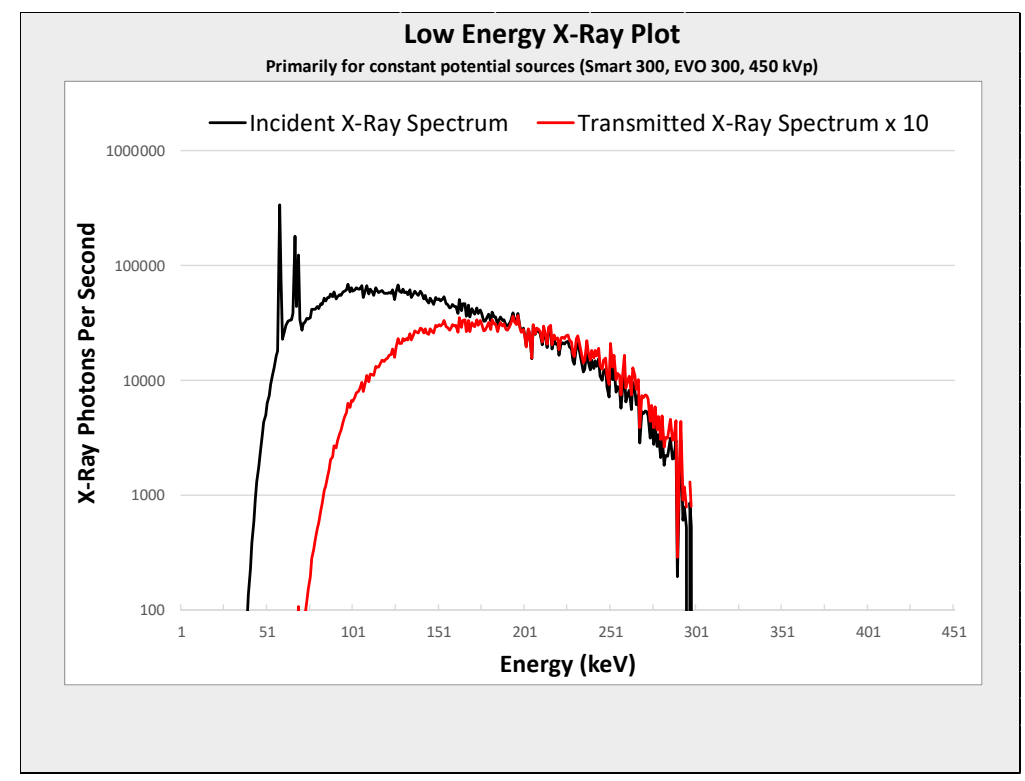

Figure 11. Example Low Energy X-Ray Plot.

There are several points to note about the plot itself. The vertical axis is number of X-ray photons per second and is logarithmically-scaled. This is done to ensure that both the incident and transmitted X-ray energy spectrum are visible on the same plot. In contrast the horizontal axis, the photon energy, is linearly scaled. The plot shows both the incident X-ray spectrum and the transmitted X-ray spectrum. The transmitted X-ray spectrum is multiplied by 10 to ensure that both spectra are visible on the plot. 


\subsubsection{High Energy X-Ray Plot}

The High Energy X-Ray Plot (Figure 12) is used to display the transmission through the object for the full energy region $(0-7.5 \mathrm{MeV})$. While the constant potential XRG energy spectra can be viewed on this plot, it is intended to be used with high-energy XRGs such as the $2.5 \mathrm{MeV}$ and $6.0 \mathrm{MeV}$ betatrons in the future. As with the Low Energy X-Ray Plot, this plot is primarily present to illustrate attenuation and beam hardening as X-rays pass through materials for the constant potential X-ray sources. The source filters are accounted for in both the incident and transmitted spectra. The detector filters are not used to calculate the spectra in this plot.

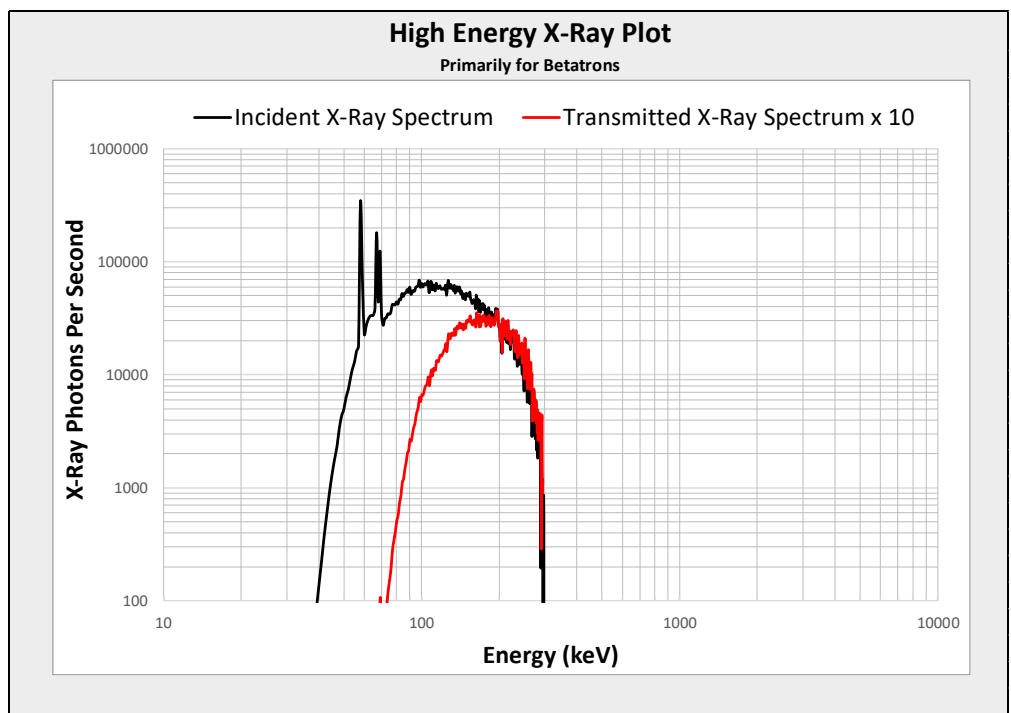

Figure 12. Example High Energy X-Ray Plot.

There are several points to note about the plot itself. The vertical axis is number of X-ray photons per second and is logarithmically-scaled. This is done to ensure that both the incident and transmitted X-ray energy spectrum are visible on the same plot. The horizontal axis, the photon energy, is also logarithmically-scaled because the Bremsstrahlung peak from high-energy XRGs is generally in the 200 $\mathrm{keV}$ range but photons with energies out to maximum kinetic energy will also be present. The plot shows both the incident X-ray spectrum and the transmitted X-ray spectrum. The transmitted X-ray spectrum is multiplied by 10 to ensure that both spectra are visible on the plot. 


\section{Verification Data}

Several verification data sets have been collected. The primary focus of these data sets has been the DRCT SMS in standard configuration with a Yxlon Smart 300 HP and a DT iHE LDA. The primary data set used for verification was a digital radiograph (DR) of a steel step wedge. The thickness of the steps in the stepwedge ranges from 0.125 inches $\left(1 / 8^{\text {th }} \mathrm{inch}\right)$ at the top to 1 inch at the bottom. The steps increase in 0.125 inches $\left(1 / 8^{\text {th }}\right.$ inch $)$ increments. A cropped version of the DR can be seen in Figure 13.

Figure 13. Cropped DR of the steel stepwedge used for verification.

\begin{tabular}{|c|c|c|c|}
\hline $\begin{array}{c}\text { Steel Step Thickness } \\
\text { (inches) }\end{array}$ & $\begin{array}{c}\text { Relative Transmission } \\
\text { from Image }\end{array}$ & $\begin{array}{c}\text { Relative Transmission } \\
\text { using Calculator }\end{array}$ & $\begin{array}{c}\text { Relative } \\
\text { Error }\end{array}$ \\
\hline 0.125 & $54.71 \%$ & $52.41 \%$ & $4.21 \%$ \\
\hline 0.250 & $32.55 \%$ & $31.27 \%$ & $3.95 \%$ \\
\hline 0.375 & $20.47 \%$ & $19.61 \%$ & $4.22 \%$ \\
\hline 0.500 & $13.13 \%$ & $12.66 \%$ & $3.55 \%$ \\
\hline 0.625 & $8.87 \%$ & $8.34 \%$ & $5.99 \%$ \\
\hline 0.750 & $6.13 \%$ & $5.57 \%$ & $9.18 \%$ \\
\hline 0.875 & $4.47 \%$ & $3.77 \%$ & $15.67 \%$ \\
\hline 1.000 & $3.51 \%$ & $2.57 \%$ & $26.74 \%$ \\
\hline
\end{tabular}

Table 1. Relative transmission from a DR compared to calculations.

Relative transmission calculated using a DR and the Steel Equivalency Calculator can be seen in Table 1. The calculator does a remarkable job of predicting the transmission when the equivalent steel thickness is below 0.750 inches (3/4 inch). It is within $10 \%$ of the actual values. However, this figure 
rapidly diverges for thicknesses exceeding 0.750 inches $(3 / 4 \mathrm{inch})$. The reason for this is discussed further in section 5 .

Verification data sets have been collected with other systems, primarily LIXS. LIXS has been tested in several configurations, including a (1) Yxlon Smart 300 HP and DT iHE2 LDA and (2) JME 6.0 MeV Betatron with both a GE DXR250U-W DDA and a DT iHE2 LDA. The LIXS data sets have yet to be processed.

\section{Path Forward}

Further work is required to both (1) improve the current iteration of the Steel Equivalency Spreadsheet and associated models and (2) verify the full complement of RCMD imaging systems. Improvements to the computation algorithm are required to address shortcomings when calculating relative transmission through thick, dense items (steel equivalent thickness $>0.750$ inches). The response of other detectors, such as DDAs, and other XRGs than those addressed in this report need to be adequately modeled and characterized (i.e. XRS4, 2.5 MeV Betatron, 6.0 MeV Betatron).

Firstly, the divergence when calculating relative transmission through thick, dense items (steel equivalent thickness $>0.750$ inches) needs to be addressed. The primary cause of the divergence in the verification data comes from the model used to calculate transmission, Beer's Law. Beer's law assumes that all incident photons are either absorbed (attenuated) or transmitted. This is not true, especially for higher energy $(>100 \mathrm{keV})$ photons, when looking at the materials of interest $(Z \leq 26)$. Incoherent scattering dominates above $100 \mathrm{keV}$, meaning that a majority of these photons are scattered and converted into lower energy photons through electronic interactions. This increases the population of lower energy photons compared to predictions by Beer's law. These lower energy photons have a higher absorption probability in the detector, increasing both the signal measured by the detector and relative transmission through the object. Either (1) a new model and updated computation method or (2) a full simulation of the imaging system (XRG, object, detector) is required for increased precision and accuracy. A full simulation of the imaging system is cost and time prohibitive with respect to both the level of effort and required computational resources. Alternative methods that calculate the conversion probability are being pursued.

To address the second point, improved models need to be generated. The current modeling software, pyPENELOPE, is reaching the end of its utility. When modeling XRGs, it can only accommodate planar stacks of materials with an angled electron beam input. Current candidate successor programs include MCNP and GEANT4. Both these programs allow for arbitrary input geometries and transient measurements that will be more indicative of the XRGs used in DRCT operations. While MCNP does not account for relativistic effects, it is being pursued due to is prevalence at INL and throughout the DOE complex. GEANT is less "user-friendly" but is necessary for high-energy XRG modeling because it accounts for relativistic effects. Both programs can be operated on the INL High Performance Computing (HPC) clusters. Use of INL HPC will expedite modeling efforts as models will be completed in hours as opposed to taking one week to complete.

\section{Summary}

The Steel Equivalency Spreadsheet was created for RCMD as a robust, user-friendly method for:

1. Determining if it is possible to image an object.

2. Reducing time needed when selecting equipment and preparing for field imaging activities.

3. Reducing the amount of equipment taken for field imaging activities.

4. Determining a starting point for exposure settings prior to imaging an object. 
5. Reducing the amount of dose deposited to complete an imaging operation (in the spirit of As Low As Reasonably Achievable, ALARA).

The spreadsheet was developed using various physical models to account for different phenomena. Future implementations aim to expand beyond the DRCT SMS in standard configuration and include high energy XRGs (Betatrons) and sub-MeV pulsed XRGs (XRS4). Alternative computational methods that compensate for incoherent scattering when calculating relative transmission are also being pursued. Ultimately the spreadsheet exceeded the developmental goal of having less than $10 \%$ average error when comparing calculations to real-world images. 


\section{References}

[1] NIST XCOM: Photon Cross Sections Database. https://www.nist.gov/pml/xcom-photon-crosssections-database

[2] Landström, Eric. "Material Identification using Multiple X-Ray Absorptiometry." (2015).

[3] Moszynski, M., et al. "CdWO4 crystal in gamma-ray spectrometry." IEEE Symposium Conference Record Nuclear Science 2004. Vol. 2. IEEE, 2004.

[4] Efficiency Calculations for Selected Scintillators. Saint-Gobain Crystals.

https://www.crystals.saint-gobain.com/sites/imdf.crystals.com/files/documents/efficiency-calculations.pdf 